\title{
Residual Stress Removal Under Pulsed Electric Current
}

\author{
Siqi Xiang ${ }^{1} \cdot$ Xinfang Zhang ${ }^{1}$
}

Received: 13 May 2019 / Revised: 15 June 2019 / Published online: 16 August 2019

(c) The Chinese Society for Metals (CSM) and Springer-Verlag GmbH Germany, part of Springer Nature 2019

\begin{abstract}
The effect of a pulsed electric current on the residual stress evolution of metal materials has been investigated. It was found that the surface and internal residual stresses in the as-quenched samples were reduced dramatically by electropulsing. A large number of experimental data show that the residual stress reduction is proportional to the initial residual stress and related to the material properties and electropulsing parameters. Under the combined actions of drift electrons, Joule heating, and residual stress, the dislocation mobility was enhanced, resulting in plastic strain and the decrease in residual stress. Drift electrons played a unique role in the electropulsing treatment, acting as an additional force pushing dislocations forward. The dislocations ultimately accumulated at a grain boundary, forming a parallel arrangement. Finally, the phenomenological equation of the residual stress evolution under electropulsing was derived from the experimental data.
\end{abstract}

Keywords Electroplastic $\cdot$ Residual stress $\cdot$ Dislocation movement $\cdot$ Phenomenological equation

\section{Introduction}

Residual stress remained in a body is stationary and at equilibrium with its surroundings. Indeed, unexpected failure is often due to combination of service stresses with residual stress therefore greatly shorten the component life [1]. In practice, it is not likely that any manufactured component is entirely free from residual stresses introduced during processing [2]. Therefore, the removal of residual stress is an important issue, especially in aerospace, nuclear, and other critical engineering industries [3].

When electropulsing is applied to metals undergoing deformation, the deformation resistance is reduced dramatically, and the plasticity increases significantly. The influence of electropulsing on the plastic flow is called the electroplastic effect [4] and is widely applied in manufacturing processes such as drawing and rolling. The electroplastic effect makes it possible to reduce the force parameters of the metal-forming process so as to obtain wires, strips, and other products with the desired physicomechanical characteristics

Available online at http://link.springer.com/journal/40195

Xinfang Zhang

xfzhang@ustb.edu.cn

1 State Key Laboratory of Advanced Metallurgy, School of Metallurgical and Ecological Engineering, University of Science and Technology Beijing, Beijing 100083, China
[5]. The electroplastic effect was discovered by Troitskii and Lichtman [6], who proposed that moving electrons in a metal crystal may interact with dislocations. Conrad and coworkers systematically investigated the decrease in flow stress when current pulse was applied to the metals $\mathrm{Al}, \mathrm{Cu}, \mathrm{Ti}, \mathrm{Ni}, \mathrm{Fe}$, $\mathrm{W}$, and $\mathrm{Nb}$ under uniaxial tensile deformation at a constant strain rate [7-13]. They found that the flow stress decreased sharply when electropulsing with the order of $10^{3}-10^{4} \mathrm{~A} /$ $\mathrm{mm}^{2}$ was applied for $60 \mu \mathrm{s}$. The stress reduction $\Delta \sigma_{\mathrm{f}}$ had two components, $\Delta \sigma_{\mathrm{E}}$ and $\Delta \sigma_{\mathrm{p}}$, which reflected reversible and irreversible strains, respectively. The irreversible strains corresponded to plastic deformation of the material [11]. Tang et al. [14-20] and other investigators [21-27] recently conducted a series of extensive studies on engineering applications of the electroplastic effect.

Because electropulsing can lead to plastic flow of metals, elimination of residual stress by electropulsing seems to be feasible. The relationship between electropulsing treatment and the residual stress evolution has been studied for over four decades. Pan et al. [28], Ragozin and Polianin [26], Stepanov et al. [27, 29, 30], Wang et al. [31], and He et al. [32] conducted a series of studies on the effect of electropulsing on the residual stress in various metal materials. These investigations showed that the residual stress was indeed reduced by electropulsing. However, some researchers remain skeptical about the effect of electropulsing on the residual stress. Huang [33] found that the residual stress in 
$16 \mathrm{Mn}$ steel plates increased by $20 \%$ on average after electropulsing treatment at a current density of $10 \mathrm{~A} / \mathrm{mm}^{2}$. Therefore, the mechanism of and law governing the elimination of residual stress by electropulsing are still not clear, and whether this method can remove residual stress stably is still controversial. Therefore, it is very important for its industrial application to clarify this issue.

The objective of this investigation is to explore the feasibility of eliminating residual stress by electropulsing. On the basis of a large number of experimental data, stable relaxation of residual stress by high current density electropulsing was proved to be feasible. A phenomenological model of the electroplastic effect on the residual stress was proposed.

\section{Experimental}

\subsection{Initial Sample Preparation}

The experimental material used in this study was $\mathrm{X} 80$ pipeline steel containing $0.058 \mathrm{C}, 0.22 \mathrm{Si}, 1.70 \mathrm{Mn}, 0.23 \mathrm{Cr}$, $0.22 \mathrm{Ni}, 0.25 \mathrm{Mo}, 0.073 \mathrm{Nb}$, and $0.014 \mathrm{Ti}$. After being cut to a size of $1.5 \mathrm{~mm} \times 10 \mathrm{~mm} \times 30 \mathrm{~mm}$, all the samples were polished by 1500 mesh sandpaper and austenitized at 880 , 900,920 , or $950^{\circ} \mathrm{C}$ for $5,6,7,8,10$, or 12 min before being water quenched to obtain different magnitudes of residual stress. During water quenching, the samples underwent the martensite phase transformation, which resulted in phase transition stress. Simultaneously, thermal stress was produced owing to the difference in cooling speed in different depths of the samples. However, the phase transition stress was dominant in the water-quenched samples, resulting in tensile stress on the surface and compressive stress in the core. In addition, the temperature difference between the surface and core is the largest for these samples. Therefore, their deformation difference is the largest, resulting in a large residual stress at the surface and in the core.

\subsection{Residual Stress Analysis}

After the surfaces of the samples were chemically polished, the surface residual stress along the long axis in the centers of the samples (the diameter of the measured area was $2 \mathrm{~mm}$ ) was measured by an X-ray diffractometer (X-350A) using the fixed $\psi$ method $[1,2,34,35]$ before and after electropulsing treatment. The following diffraction conditions were adopted: (a) The diffraction anode was made of $\mathrm{Cr}$, (b) $K_{\alpha}$ radiation was used, (c) the diffraction lattice planes were the $\{221\}$ family of crystal lattice planes, (d) the diffraction angle was $151^{\circ}-162^{\circ}$, (e) the diffraction spot diameter $\Phi$ was $2 \mathrm{~mm}$, (f) the scanning step was $0.1^{\circ},(\mathrm{g})$ the count time per step was $0.5 \mathrm{~s}$, (h) the scanning voltage was $20 \mathrm{kV}$, (i) the scanning current was $5 \mathrm{~mA}$, and (j) the elastic constant was
$316 \mathrm{MPa} /{ }^{\circ}$. To ensure accurate measurement, eight fixed values of $\psi$ (the angle between the normal of the sample and the normal of the diffracting lattice planes) were adopted: $0^{\circ}$, $8^{\circ}, 16^{\circ}, 24.2^{\circ}, 30^{\circ}, 35.3^{\circ}, 40^{\circ}$, and $45^{\circ}$. To avoid the effect of natural aging, the residual stress was measured 1 day after water quenching. Furthermore, the samples were treated by electropulsing within 10 days, and the residual stress was measured immediately after the electropulsing treatment.

The internal residual stress at different depths in the samples was measured after electrolytic polishing to remove the surface layer. The electrolyte consisted of a mixture of $90 \%$ ethanol and $10 \%$ perchloric acid at room temperature; polishing was performed for $5 \mathrm{~min}$ at an applied voltage of $30 \mathrm{~V}$ and applied current of $2 \mathrm{~A}$. The residual stresses were measured immediately after each electrolytic polishing process.

\subsection{Electropulsing Treatment}

The electropulsing equipment consisted of several $1200 \mu \mathrm{F}$, $1800 \mathrm{~V}$ capacitors that were connected in series or in parallel to achieve different working current densities $J$ and pulse widths $w$. The samples were clamped between two copper electrodes. Further, a Rogowski coil-based system was used to detect the electric pulse, and its output was examined using a digital storage oscilloscope. According to the waveform recorded by the oscilloscope, the current intensity is approximately expressed as [36]

$I(t)=I_{\mathrm{m}} e^{-\alpha_{0} t} \sin \frac{2 \pi}{\tau_{0}} t$,

where $I(t)$ is the current intensity, $I_{\mathrm{m}}$ is the maximum value of the first sinusoidal wave peak, and $\alpha_{0}$ is the attenuation coefficient. The waveform of the pulses is essentially that of damped oscillation. Figure 1 shows typical profiles of the

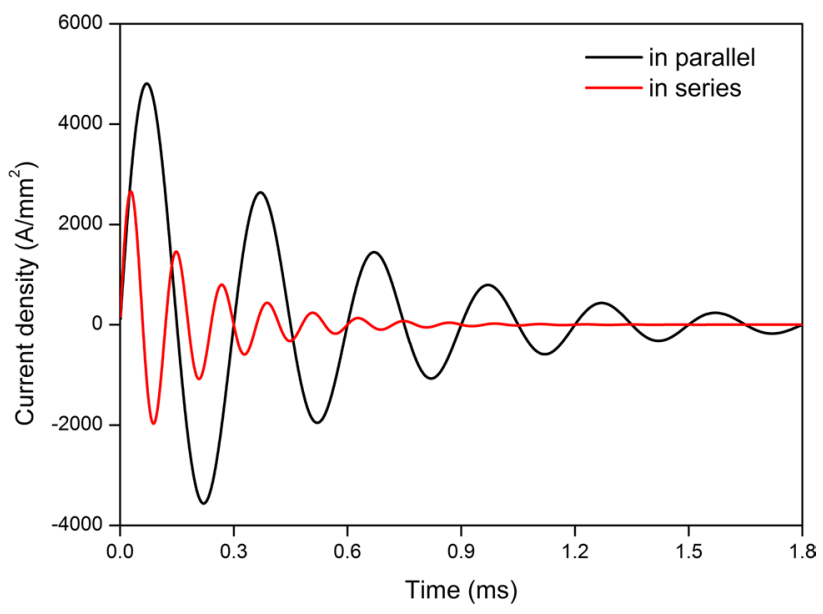

Fig. 1 Typical profiles of current density versus time for electropulses produced by capacitors connected in series and in parallel 
current density versus time for electropulsing at $I_{\mathrm{m}}=46,170$ A, $W=60 \mu \mathrm{s}, \alpha_{0}=5000$ (with the capacitors connected in series) and $I_{\mathrm{m}}=83,430 \mathrm{~A}, W=150 \mu \mathrm{s}, \alpha_{0}=2000$ (with the capacitors connected in parallel). The current density $J(t)=\frac{I(t)}{S}$, where $S=15 \mathrm{~mm}^{2}$ is the cross-sectional area of the samples. A negative current density indicates that the current direction is opposite to the initial current direction. When the capacitors were connected in parallel, the single oscillation time and total oscillation time were much higher than that when the capacitors were connected in series.

\section{Results and Discussion}

Single electropulses produced by capacitors connected in series with different current densities ranging from 1469 to $3078 \mathrm{~A} / \mathrm{mm}^{2}$ were applied to more than ten samples with different initial residual stresses ranging from 135 to $435 \mathrm{MPa}$. However, the residual stress was not eliminated by the electropulse produced by capacitors connected in series. As Wang et al.'s [31] results show, when the current density was $5.5 \mathrm{kA} / \mathrm{mm}^{2}$, the surface residual stress reduction rate was as low as $50 \%$. In addition, the effect of electropulsing at current densities below $5.5 \mathrm{kA} / \mathrm{mm}^{2}$ on the residual stress was not reported in [31], which implies that a lower current density would have little effect on the residual stress. That is, there appears to be a critical current density below which electropulsing cannot eliminate residual stress. In the present investigation, the current density was only $3 \mathrm{kA} / \mathrm{mm}^{2}$ when the capacitors were connected in series. Therefore, the residual stress could not be eliminated by electropulsing by capacitors connected in series, given this low current density.

When the capacitors were connected in parallel, the current density ranged from 3942 to $5562 \mathrm{~A} / \mathrm{mm}$. As shown in Fig. 2, after the electropulsing treatment, the residual stresses of 70 samples with different initial residual stresses ranging from 34 to $485 \mathrm{MPa}$ were dramatically reduced. With increasing current density, the residual stress reduction increased gradually. When the current density reached 5562 $\mathrm{A} / \mathrm{mm}^{2}$, the residual stress removal rate was over $80 \%$, and the residual stress was almost completely eliminated.

As shown in Fig. 3, the initial surface residual stresses of all the samples were approximately $380 \mathrm{MPa}$. It is clear that
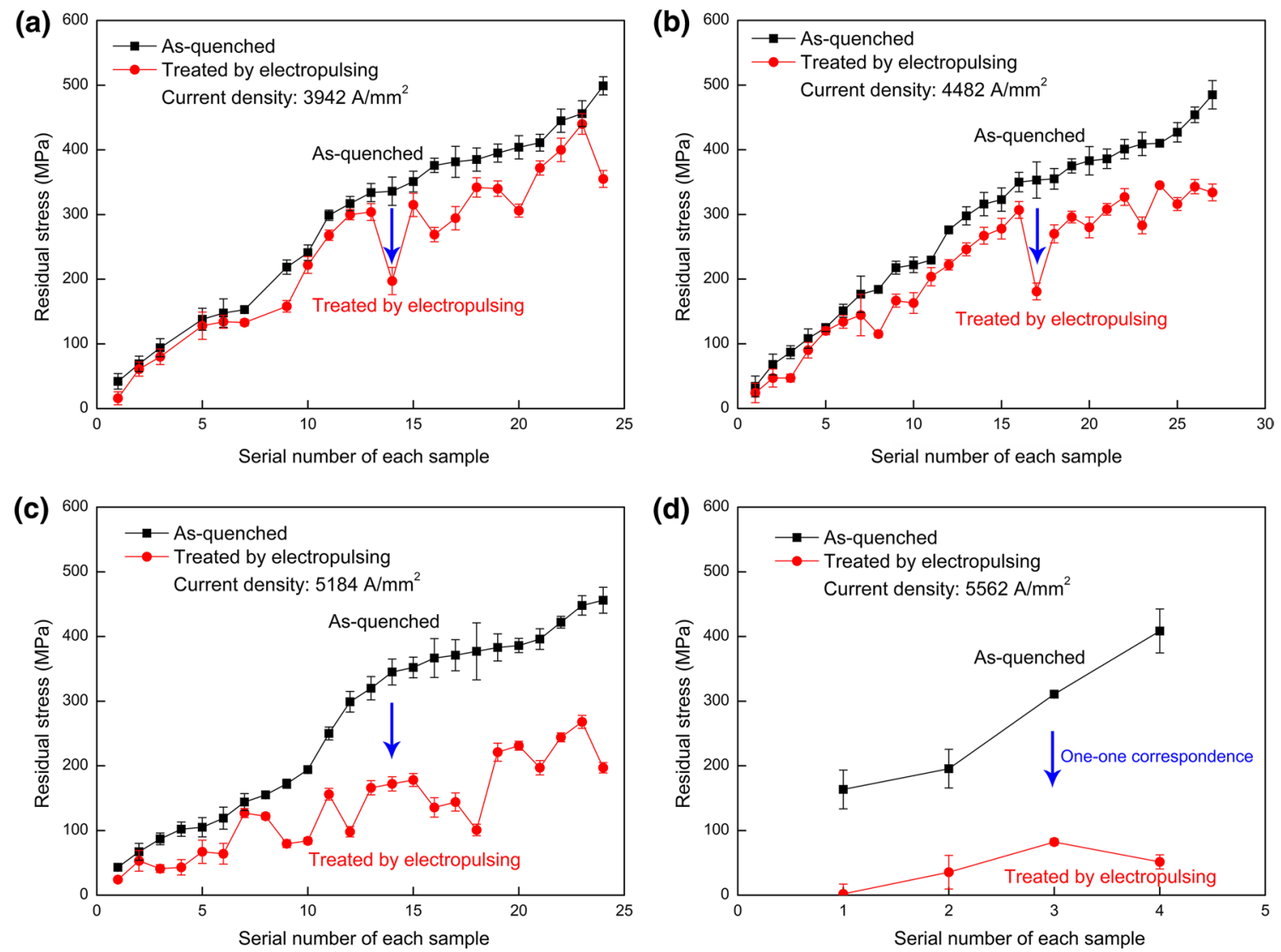

Fig. 2 Surface residual stress of approximately 70 as-quenched samples before and after electropulsing treatment at different current densities: a $3942 \mathrm{~A} / \mathrm{mm}^{2}$, b $4482 \mathrm{~A} / \mathrm{mm}^{2}$, c $5184 \mathrm{~A} / \mathrm{mm}^{2}$, d $5562 \mathrm{~A} / \mathrm{mm}^{2}$. Square and circular points with the same serial number correspond to the same sample before and after electropulsing treatment, respectively 


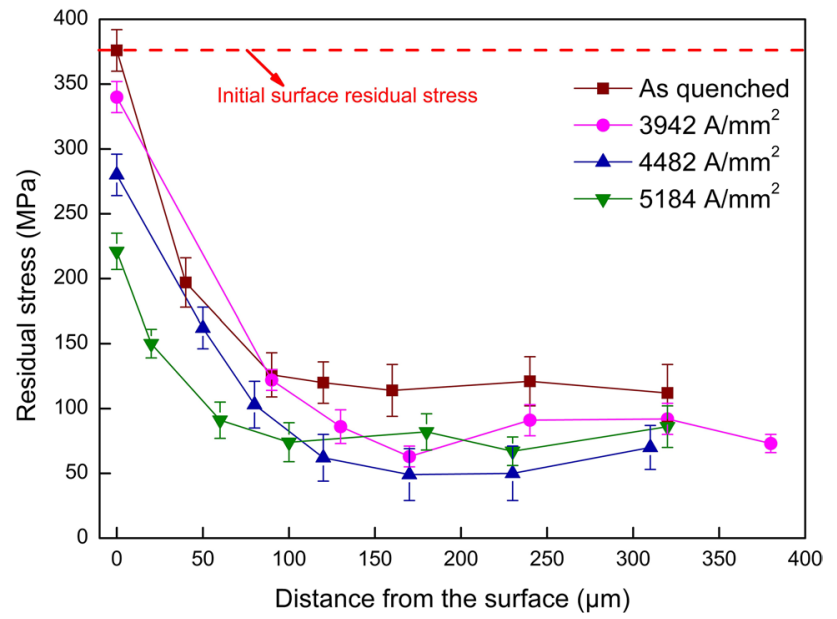

Fig. 3 Residual stresses of the as-quenched sample and electropulsed samples at different depths

the residual stresses at different depths in the samples were also reduced by the electropulsing treatment, which implies that electropulsing can eliminate residual stress throughout the sample rather than only on the surface. In addition, the residual stresses at different depths were measured by electropolishing layer by layer. Note that during surface polishing, the gradual release of residual stress will distort the measured value of the internal residual stress, especially when the measurement depth exceeds one-quarter of the sample thickness ( $375 \mu \mathrm{m}$ in this experiment).

As shown above, electropulsing successfully eliminated the residual stress in the entire sample, and the residual stress was completely eliminated when the current density was sufficiently high. After electropulsing treatment at the same current density, the residual stress reduction $\Delta \sigma$ increased linearly with increasing initial residual stress $\sigma_{0}$, as shown in Fig. 4.

In addition, the $y$-axis intercept of the fitting line is negligible relative to the total residual stress elimination, and thus, the reduction in residual stress $\Delta \sigma$ is proportional to the initial residual stress $\sigma_{0}$, giving

$\Delta \sigma=k \sigma_{0}$,

where $k$ is the slope of the fitting line.

The slope $k$ is clearly also the residual stress reduction rate; i.e., when the electropulsing parameters are constant, the residual stress reduction rate is constant.
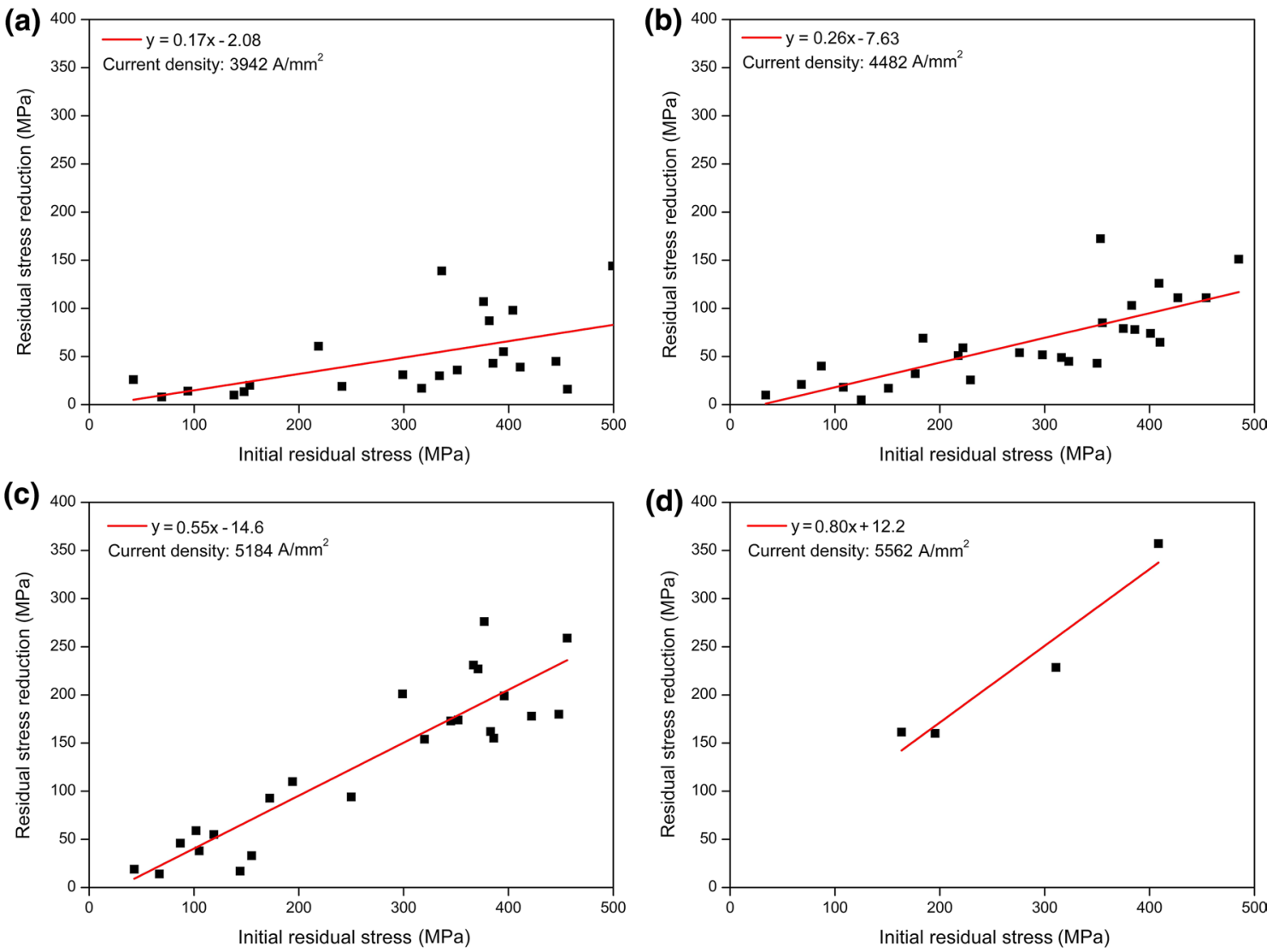

Fig. 4 Residual stress reduction in the samples with initial residual stresses ranging from 50 to $500 \mathrm{MPa}$ after electropulsing treatment at different current densities: a $3942 \mathrm{~A} / \mathrm{mm}^{2}$, b $4482 \mathrm{~A} / \mathrm{mm}^{2}$, c $5184 \mathrm{~A} / \mathrm{mm}^{2}$, d $5562 \mathrm{~A} / \mathrm{mm}^{2}$ 


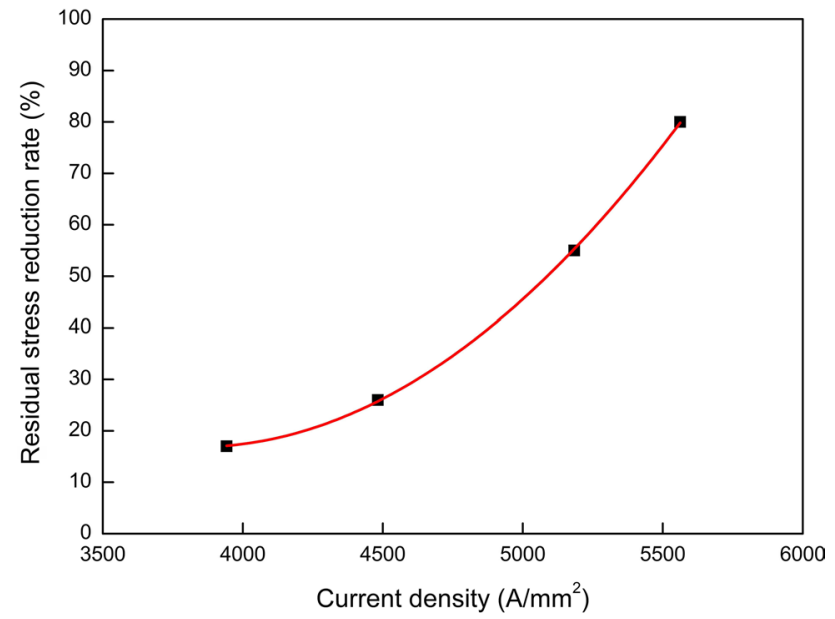

Fig. 5 Residual stress reduction rate versus current density

Figure 5 shows the relationship between the residual stress reduction rate $k$ and the current density $J$. Note that the residual stress reduction rate increases with increasing current density, showing a quadratic-like relationship.

In this investigation, the plastic strain $\Delta \varepsilon_{\mathrm{p}}$ is proportional to the change in the residual stress $\Delta \sigma$ of the samples, giving

$\Delta \sigma=E \Delta \varepsilon_{\mathrm{p}}$,

where $E$ is the Young's modulus.

The residual tensile stress in the surface layer of the sample is essentially the same as the tensile stress applied to the sample during uniaxial tensile deformation. That is, under electropulsing, the response of the material to electropulsing is consistent, regardless of the external stress or residual stress exerted upon it. However, the residual stress at different depths in the samples is different, which suggests that the overall deformation behavior of the samples is not the same as that produced by the uniaxial tensile process under electropulsing, as the residual stress inside the samples would influence the surface residual stress. At the same current density, because the residual stress on the surface is the largest, the plastic strain on the surface is clearly also the largest. Because the plastic strain in the samples at different depths is less than that on the surface (the strain is proportional to the initial stress), the internal structure would block the surface strain, and consequently the actual plastic strain would be less than the nominal plastic strain. Although this effect is not negligible, it is small. By comparing the present experimental results with those of Conrad and Okazaki [11] and ignoring the effect of the inner structure on the surface mentioned above, we find that the change in residual stress $\Delta \sigma$ is equivalent to the irreversible stress decrease $\Delta \sigma_{\mathrm{p}}$ of metals treated by electropulsing and undergoing uniaxial tensile deformation at a constant strain rate.
Hence, the plastic strain $\Delta \varepsilon_{\mathrm{p}}$ during residual stress elimination by electropulsing is equivalent to the irreversible plastic strain under uniaxial tension. Because the residual stress reduction rate and current density $J$ have a quadraticlike relationship, the relationship between the residual stress reduction $\Delta \sigma$ and current density $J$ is quadratic-like when the initial stress is constant (where the initial residual stress corresponds to the initial stress in this investigation, and the flow stress before the current pulse corresponds to the initial stress in the uniaxial tension process). That is, the relationship between the plastic strain $\Delta \varepsilon_{\mathrm{p}}$ and current density $J$ is quadratic-like when the initial stress is constant.

Figure 6 shows the experimental data obtained in this investigation, and those reported by Conrad are also shown [13]. The solid line in Fig. 6 is the experimental results of the relationship between the plastic strain $\Delta \varepsilon_{\mathrm{p}}$ and the current density for six groups of the samples treated by electropulsing (which was obtained from the residual stress reduction using Eq. (3). The initial residual stress of the samples in each group was the same. As mentioned above, the actual surface plastic strain would be less than the nominal plastic strain, but the difference would be small. Further, when the initial residual stress was the same (regardless of its value), the relationship between the residual stress reduction rate and the current density was quadratic-like which was consistent with that shown in Fig. 5. The dashed lines in Fig. 6 show the effect of the current density on the irreversible strains for current pulses applied to metals that had been deformed by uniaxial tension in the work of Sprecher et al. [11]. The relationship between $\Delta \varepsilon_{\mathrm{p}}$ and the current density $J$ obtained in this study was clearly the same as these obtained by Conrad et al. [13] Note that the different positions of the

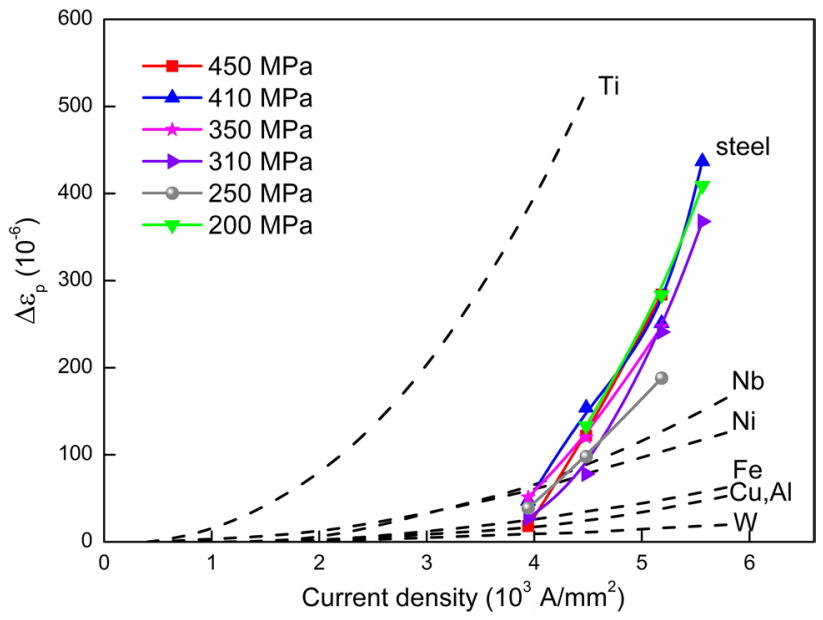

Fig. 6 Plastic strain $\Delta \varepsilon_{\mathrm{p}}$ versus current density. The solid line is the experimentally obtained relationship between the plastic strain and the current density of six groups of samples, where the initial residual stress of the samples in each group was the same. The dashed lines are the experimental results of Conrad [13] 
curves obtained in this study and those obtained by Conrad result from differences in the electropulsing waveforms (pulse width, number of oscillation peaks, and pulse frequency) and the type of metal treated. In conclusion, the agreement between the results of this investigation and Conrad's results further confirmed that the relationship between the residual stress reduction rate and the current density is quadratic-like.

Natural aging is known to reduce the residual stress. However, natural aging can reduce the residual stress only by $10 \%$ [35]. In this experiment, to exclude the effect of natural aging, we took a quenched sample, whose residual stress was measured immediately after water quenching, at room temperature for more than 10 days (all the other samples were treated by electropulsing within 10 days after water quenching) and then measured its residual stress. We found no difference between the residual stress values before and after natural aging.

During the electropulsing treatment, the direct effect of electropulsing is accompanied by side effects such as Joule heating, pinch, and skin effects. The contribution of these side effects to the electroplastic effect is an important issue but is still controversial [4]. Okazaki et al. [37] evaluated the contributions of the skin and pinch effects to the electroplastic effect and proposed that they do not greatly affect the plastic strain of metals and cannot reduce the residual stress to the degree observed in this study.

The temperature increase $\Delta T$ during electropulsing is given by [38]

$\Delta T=\int_{0}^{t} \rho J(t)^{2}\left(C_{\mathrm{p}} d\right)^{-1}$,

where $\rho$ is the resistivity, the current density $J(t)\left[=\frac{I(t)}{S}\right], S$ $\left(=15 \mathrm{~mm}^{2}\right)$ is the cross-sectional area, $C_{\mathrm{p}}$ is the specific heat, and $d$ is the sample density. In this investigation, the value of $\Delta T_{\max }$ calculated for $J_{\max }=5562 \mathrm{~A} / \mathrm{mm}^{2}$, $\rho=2.746 \times 10^{-5} \Omega \mathrm{cm}, C_{\mathrm{p}}=540 \mathrm{~J} /(\mathrm{kg} \mathrm{K})$, and $d=8 \mathrm{~g} / \mathrm{cm}^{3}$ was $240{ }^{\circ} \mathrm{C}$. As Sprecher et al. [11] proposed, the plastic flow $\Delta \varepsilon_{\mathrm{p}}$ due to Joule heating was only on the order of $10^{-9}$ for $\mathrm{Cu}$ treated by a single current pulse of $J=5500 \mathrm{~A} / \mathrm{mm}^{2}$ for $60 \mu \mathrm{s}$. Their results indicate that Joule heating did not contribute significantly to the plastic strain component $\Delta \varepsilon_{\mathrm{p}}$ of the load drop.

Note that, to verify the effect of temperature on the plastic strain, the as-quenched samples were treated by continuous low current density electropulsing [4]. The residual stress of the first as-quenched sample changed from 368 to $363 \mathrm{MPa}$ after treatment by continuous electropulsing for $1 \mathrm{~min}$ at a maximum current density of $67 \mathrm{~A} / \mathrm{mm}^{2}$, frequency of $500 \mathrm{~Hz}$, and the pulse duration of $60 \mu \mathrm{s}$. The residual stress of the second quenched sample changed from 363 to $371 \mathrm{MPa}$ after treatment by continuous electropulsing for
1 min at a maximum current density of $100 \mathrm{~A} / \mathrm{mm}^{2}$, frequency of $500 \mathrm{~Hz}$, and the pulse duration of $60 \mu \mathrm{s}$. The temperature during continuous electropulsing was above $320^{\circ} \mathrm{C}$ (as measured by a thermocouple at the end of the electropulsing). The result suggested that Joule heating was not the decisive factor in the elimination of residual stress; i.e., Joule heating cannot result in plastic deformation in such a short time. In addition, this comparison test also ruled out the possibility that the higher temperature increase at defects would lead to dislocation slip during the electropulsing treatment, in which process cannot be examined by temperature contrast tests in a muffle furnace. Note that the continuous electropulsing treatment did not reduce the residual stress because the current density was too low.

Because the side effects do not significantly affect the plastic strain of the samples, we next consider the effect of drift electrons on the plastic strain of the samples. As proposed by Conrad and coworkers [7-13], drift electrons affect dislocations. To study the effects of drift electrons on dislocations, samples were observed by transmission electron microscopy (TEM) after polishing by a double-jet electropolisher using an electrolyte consisting of a mixture of $90 \%$ ethanol and $10 \%$ perchloric acid at $-35^{\circ} \mathrm{C}$. Figure 7 shows TEM images of a quenched sample and a sample treated by electropulsing at a current density of $5184 \mathrm{~A} / \mathrm{mm}^{2}$. Both samples were observed from the [110] direction and imaged using multiple $\mathrm{g}$-vectors so that all the dislocations are visible in each image. As shown in Fig. 7a, the as-quenched sample contained many dislocation tangles. However, after the electropulsing treatment, the dislocation density clearly decreased, and the dislocations had an obvious parallel arrangement (Fig. 7b). This phenomenon was not an accidental phenomenon but was very significant, appearing in more than ten samples treated by electropulsing at different current densities.

To explore the relationship between drift electrons and the parallel dislocations, the direction of the parallel dislocations was calibrated by the trace method [39]. The sample in Fig. 8 was treated by electropulsing at a current density of $5184 \mathrm{~A} / \mathrm{mm}^{2}$. The images in Fig. 8 were taken using two different beam directions parallel to the [001] and [023] directions. The two images are therefore two different projections of the dislocations. Images of this type were used to determine the direction of the dislocation lines. The dislocation lines are $65^{\circ}$ from the horizontal in Fig. $8 \mathrm{a}$ and $58^{\circ}$ from the horizontal in Fig. 8b. According to the method described in [39], the direction of the dislocation lines in Fig. 8 was $[11 \overline{2}]$. These dislocations were thus parallel to the slip plane ( $\{1 \overline{1} 0\})$ and perpendicular to the slip direction ([111]) of the body-centered cubic crystal. In addition, the direction of the drift electrons was calibrated by marking a circular region on the sample before the TEM observation. As shown in Fig. 8a, the drift electrons were moving perpendicular 

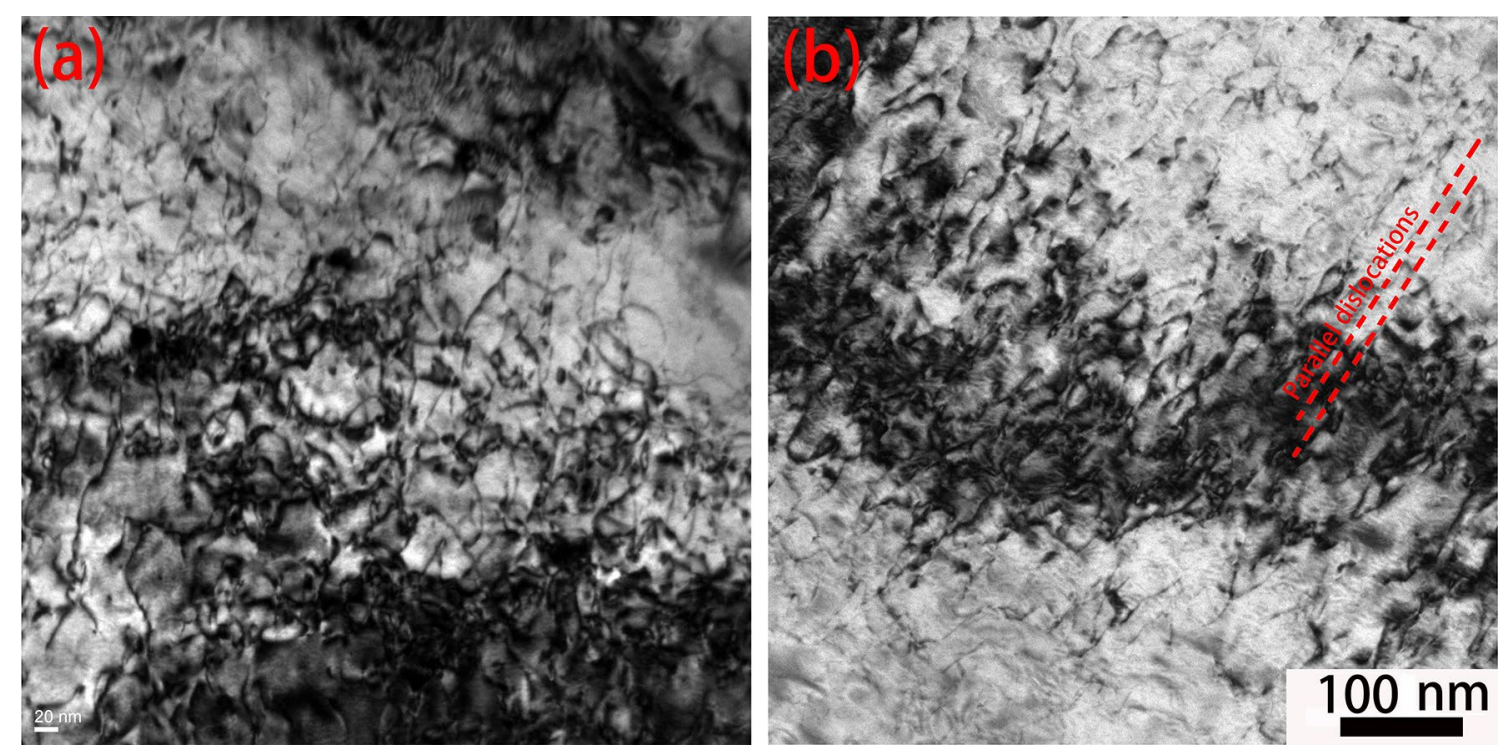

Fig. 7 TEM images of a as-quenched sample, b sample treated by electropulsing at a current density of $5184 \mathrm{~A} / \mathrm{mm}^{2}$. These samples were observed from the [110] direction

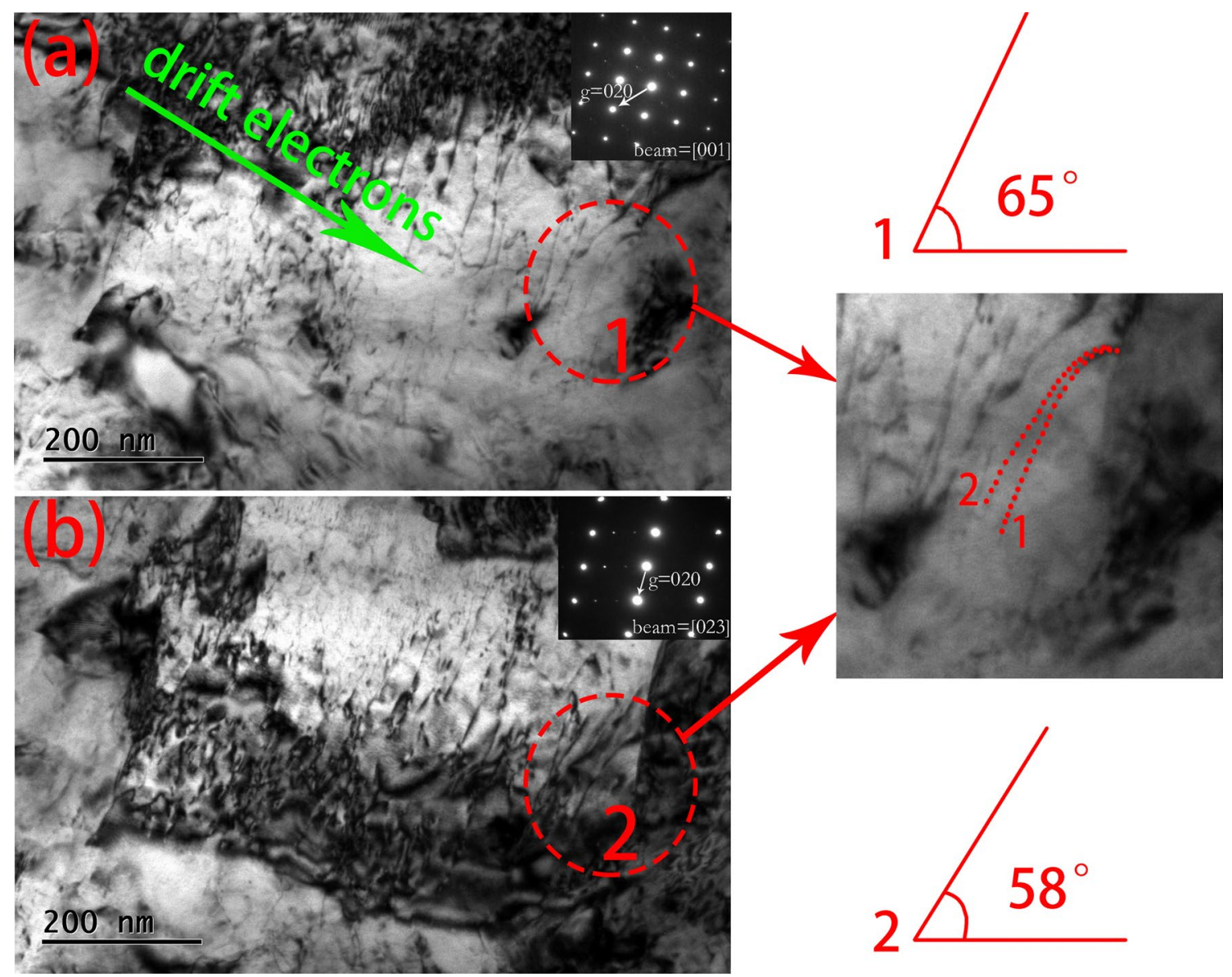

Fig. 8 Determination of dislocation line directions. a Image obtained in the [001] beam direction. The dislocation lines are $65^{\circ}$ from the horizontal. b Image obtained in the [023] beam direction. The dislocation lines are $58^{\circ}$ from the horizontal. The samples were treated by electropulsing at a current density of $5184 \mathrm{~A} / \mathrm{mm}^{2}$ 
to the dislocation lines. At the same time, the dislocations were parallel to the slip plane $(\{1 \overline{1} 0\})$ and perpendicular to the slip direction ([111]). It is reasonable to speculate that the dislocation mobility was enhanced by the action of drift electrons. Under the electron wind force, the dislocations moved forward and eventually accumulated at the grain boundary, forming a parallel arrangement. The electron wind force played a unique role in the electropulsing treatment, acting as an additional force pushing dislocations forward.

Conrad and coworkers [7-13] systematically studied the mechanism by which drift electrons affect the dislocation mobility. Considering plastic flow of metals undergoing uniaxial tensile deformation at a constant strain rate to be thermally activated, Sprecher et al. [11] gave

$\ln \left(\frac{\dot{\varepsilon_{\mathrm{ed}}}}{\dot{\dot{\varepsilon}_{1}}}\right)=\ln \left(\frac{\dot{\varepsilon_{0 \mathrm{ed}}}}{\dot{\dot{\varepsilon}_{1}}}\right)-\frac{\left(\Delta H_{\mathrm{ed}}^{*}-\Delta H^{*}\right)}{k T}+\frac{\left(\nu_{\mathrm{ed}}^{*}-v^{*}\right)}{k T}+\frac{\nu_{\mathrm{ed}}^{*} \sigma_{\mathrm{ew}}}{k T}$,

where $\dot{\varepsilon_{\text {ed }}}$ is the strain rate under electropulsing, $\dot{\varepsilon}_{0}$ is the strain rate before electropulsing, $\dot{\varepsilon}_{1}$ is a pro-exponential factor, $\Delta H^{*}$ is the activation enthalpy, $\nu^{*}$ is the activation area, $\sigma^{*}$ is the effective stress, $\sigma_{\text {ed }}$ is the additional stress acting on the dislocations owing to the force exerted by drift electrons, $k$ is the Boltzmann constant, $T$ is the temperature, and the subscript "ed" indicates the parameter for samples with the electropulsing treatment.

Equation (5) shows how drift electrons may influence other parameters relating to the thermally activated motion of dislocations in addition to exerting an electron wind force. They proposed that the contribution to $\Delta \varepsilon_{\mathrm{p}}$ in addition to the electron wind is $\alpha=a J^{2}$ (where $a$ is a constant depending on the material and temperature), which represents the first three terms of Eq. (5), and the fourth term of Eq. (5) is proportional to the current density; i.e., $\frac{v_{\mathrm{dd}}^{*} \sigma_{\mathrm{ew}}}{k T}=b J$ (where $b$ is a constant depending on the material and temperature). Hence, Eq. (5) is reduced to

$\ln \left(\frac{\dot{\varepsilon_{\mathrm{ed}}}}{\dot{\varepsilon_{0}}}\right)=a J^{2}+b J$.

In short, the effect of electropulsing on the plastic flow consists of two contributions, one proportional to $J$ and the other proportional to $J^{2}$. According to the theory of thermally activated plastic deformation, the former is associated with an electron wind force that added to the applied force by helping dislocations overcome obstacles to their motion, and the latter was associated with the effect of drift electrons on the pre-exponential term and possibly on the obstacle force-distance curve.

Combining a large number of experimental data (as shown in Fig. 2) and the theory of thermally activated motion of dislocations [11], the semiempirical equation of the residual stress reduction rate $k$ versus the current density $J$ during electropulsing is derived as

$k=A J^{2}+B J+C$.

The first term, $A J^{2}$, is the residual stress reduction caused by the effects of electropulsing on the pre-exponential and the obstacle force-distance curve. The second term, $B J$, is the residual stress reduction caused by the electron wind force. For samples with different initial residual stresses, there is a slight difference in the residual stress reduction rate after electropulsing treatment at the same current density, as shown in Fig. 6, which implies that the equation should contain a third term, $C$, corresponding to the residual stress reduction caused by the initial residual stress. Note that when the single and total oscillation times of the electropulsing were too small, the residual stress could not be reduced, even at very high current densities, implying that the pulse width and pulse frequency can also influence the residual stress reduction. Hence, the constants $A$ and $B$ should be related not only to the material properties but also to the electropulsing parameters, including the maximum current density $J$. Therefore, the residual stress reduction rate is a quadratic function of the maximum current density; i.e., when the initial residual stress is constant, the residual stress reduction is quadratic with respect to the maximum current density, as shown in Eq. (7). In addition, when the electropulsing parameters are constant, the residual stress reduction is proportional to the initial residual stress, as shown in Eq. (2). By combining Eqs. (2) and (7), the phenomenological equation of residual stress elimination by electropulsing is obtained as

$\Delta \sigma=\sigma_{0}\left(A J^{2}+B J+C\right)$.

By combining Eqs. (3) and (8), the phenomenological equation of the plastic strain caused by electropulsing is obtained as

$\Delta \varepsilon_{\mathrm{p}}=k \frac{\sigma_{0}}{E}=\left(A J^{2}+B J+C\right) \frac{\sigma_{0}}{E}$.

Equation (9) can be used to evaluate the plastic strain $\Delta \varepsilon_{p}$ that occurs when electropulsing is applied to metals undergoing uniaxial tensile deformation at a constant strain rate. Note that the constants $A$ and $B$ are determined by the electropulsing parameters and the treated metals' properties. Further, the constant $C$ in the equation is determined by the tensile stress before electropulsing.

The quantitative phenomenological equation for the electroplastic effect on the residual stress and plastic strain is given in Eqs. (8) and (9). In addition, the waveform of the pulsed discharge system is essentially a damped oscillation, and the effect of electropulsing on the plastic flow was cumulative over time. Therefore, the effects of electropulsing on the pre-exponential and the obstacle force-distance curve 
are complicated and unknown. Hence, the theoretical equation of residual stress reduction by electropulsing is difficult to derive, and further work is needed.

\section{Conclusions}

1. The residual stress within an entire quenched steel sample can be dramatically reduced by electropulsing treatment.

2. The dislocation mobility is enhanced by drift electrons. Under the electron wind force, the dislocations move forward and eventually accumulate at the grain boundary, showing a parallel arrangement.

3. According to the thermally activated plastic flow concept, the drift electrons affect the thermally activated motion of dislocations, which is proportional to the square of the current density, in addition to supplying a force exerted on the dislocations by the electron wind, which is proportional to the current density. Under the combined actions of the drift electrons, Joule heating, and residual stress, the movement of dislocations is enhanced, leading to a decrease in the residual stress. Finally, the phenomenological equation is given as $\Delta \sigma=\sigma_{0}\left(A J^{2}+B J+C\right)$.

Acknowledgements The work was financially supported by the National Natural Science Foundation of China (Nos. 51874023, 51601011 and U1860206), the Fundamental Research Funds for the Central Universities, Recruitment Program of Global Experts.

\section{References}

[1] P.J. Withers, H. Bhadeshia, Mater. Sci. Technol. 17, 355 (2001)

[2] P.J. Withers, H. Bhadeshia, Mater. Sci. Technol. 17, 366 (2001)

[3] I.C. Noyan, J.B. Cohen, Residual Stress: Measurement by Diffraction and Interpretation (Springer, Berlin, 2013)

[4] L. Guan, G. Tang, P.K. Chu, J. Mater. Res. 25, 1215 (2010)

[5] N.E. Kir'yanchev, O. Troitskii, Probl. Prochn. 5, 101 (1983)

[6] O.A. Troitsky, V.I. Likhtman, Sov. Phys. Dokl. 8, 91 (1963)

[7] K. Okazaki, M. Kagawa, H. Conrad, Scr. Mater. 12, 1063 (1978)

[8] K. Okazaki, M. Kagawa, H. Conrad, Scr. Mater. 13, 277 (1979)

[9] K. Okazaki, M. Kagawa, H. Conrad, Scr. Mater. 13, 473 (1979)

[10] H. Conrad, K. Okazaki, Kentucky Univ. Lexington, Department of Metallurgical Engineering and Materials Science (1979)
[11] A.F. Sprecher, S.L. Mannan, H. Conrad, Acta Mater. 34, 1145 (1986)

[12] H. Conrad, Mater. Sci. Eng. A 287, 276 (2000)

[13] H. Conrad, Mater. Sci. Eng. A 322, 100 (2002)

[14] X. Li, Y. Ye, R. Zhang, S.Z. Kure-Chu, G. Tang, Mater. Sci. Eng. A 742, 722 (2019)

[15] Y. Ye, S.Z. Kure-Chu, Z. Sun, X. Li, H. Wang, G. Tang, Mater. Des. 149, 214 (2018)

[16] Z. Xu, H. Wang, Z. Sun, Y. Ye, G. Tang, Mater. Sci. Technol. 33, 1454 (2017)

[17] H. Wang, G. Song, G. Tang, Surf. Coat. Technol. 282, 149 (2015)

[18] R.F. Zhu, J.N. Liu, G.Y. Tang, S.Q. Shi, M.W. Fu, Z.T.H. Tse, J. Alloys Compd. 584, 225 (2014)

[19] Y.D. Ye, X.P. Li, Z.Y. Sun, H.B. Wang, G.Y. Tang, Acta Metall. Sin. (Engl. Lett.) 31, 1265 (2018)

[20] X.P. Li, S.Z. Kure-Chu, T. Ogasawara, H. Yashiro, H.B. Wang, Z.Z. Xu, G.Y. Tang, Acta Metall. Sin. (Engl. Lett.) 31, 1258 (2018)

[21] J.Y. Gao, X.B. Liu, H.F. Zhou, X.F. Zhang, Acta Metall. Sin. (Engl. Lett.) 31, 1240 (2018)

[22] Y.H. Zhu, C.M. Luk, Acta Metall. Sin. (Engl. Lett.) 31, 1361 (2018)

[23] Z. Sun, H. Wang, Y. Ye, Z. Xu, G. Tang, Int. J. Adv. Manuf. Technol. 95, 2835 (2018)

[24] B. Kinsey, G. Cullen, A. Jordan, S. Mates, CIRP Ann. 62, 279 (2013)

[25] H. Xie, X. Dong, K. Liu, Z. Ai, F. Peng, Q. Wang, J. Wang. Mater. Sci. Eng. A 637, 23 (2015)

[26] Y.I. Ragozin, I.V. Polianin, in Proceedings of the Fourth International Conference on Residual Stresses, vol. 926 (1994)

[27] G.V. Stepanov, A.I. Babutskii, I.A. Mameev, M. Ferraris, V. Casalegno, M. Salvo, Strength Mater. 40, 452 (2008)

[28] L. Pan, B. Wang, Z. Xu, J. Alloys Compd. 792, 994 (2019)

[29] G. Stepanov, A. Babutsky, L. Kruszka, Mater. Sci. Forum 638, $2429(2010)$

[30] G.V. Stepanov, A.I. Babutskii, I.A. Mameev, Strength Mater. 41, $623(2009)$

[31] J. Wang, X. He, B. Wang, J. Guo, Chin. J. Mater. Res. 21, 41 (2007)

[32] W.L. He, BGu Pan, Mater. Sci. Eng. A 662, 404 (2016)

[33] X. Huang, Mater. Sci. Eng. A 528, 6287 (2011)

[34] N.S. Rossini, M. Dassisti, K.Y. Benyounis, A.G. Olabi, Mater. Des. 35, 572 (2012)

[35] R. Gou, Y. Zhang, X. Xu, L. Sun, Y. Yang, NDT E Int. 44, 387 (2011)

[36] Z. Wang, H. Song, J. Alloys Compd. 470, 522 (2009)

[37] K. Okazaki, M. Kagawa, H. Conrad, Mater. Sci. Eng. 45, 109 (1980)

[38] H. Conrad, A.F. Sprecher, in Dislocations in Solids, ed. by F.R.N. Nabarro (Elsevier, Amsterdam, 1989), p. 497

[39] Y.L. Wei, A. Godfrey, W. Liu, Q. Liu, X. Huang, N. Hansen, G. Winther, Scr. Mater. 65, 355 (2011) 